\title{
Defective MHC class I antigen surface expression promotes cellular survival through elevated ER stress and modulation of p53 function
}

\author{
K Sabapathy ${ }^{\star, 1,2,3}$ and SY Nam ${ }^{1}$
}

Defects in Major Histocompatibility class I cell surface expression is thought to allow escape of tumor cells from immune surveillance. Hitherto, it is unclear whether this deficiency confers immune-independent survival advantage. We show here that class I cell surface expression deficiency due to defects in $\beta 2$ microglobulin or the transporter-associated with antigen processing (TAP) results in resistance to apoptosis in response to various cytotoxic signals. Reduced apoptosis correlated with altered p53 activation, which was due to compromised nuclear translocation of p53. Binding of p53 to glycogen synthase kinase$3 \beta$ (GSK3 $\beta$ ), which is known to phosphorylate and lead to cytoplasmic sequestration of p53, was enhanced in these cells. Consistently, endoplasmic reticulum (ER) stress, which promotes binding of p53 to GSK3 $\beta$ was constitutively elevated in the absence of class I cell surface expression. Taken together, the results suggest a non-immunological causal role for defective class I cell surface expression in regulating cellular survival in a p53-dependent manner, through the upregulation of ER stress, which could be another mechanism leading to carcinogenesis.

Cell Death and Differentiation (2008) 15, 1364-1374; doi:10.1038/cdd.2008.55; published online 30 May 2008

The major histocompatibility complex (MHC) class I antigens are generally ubiquitously expressed and provide the identity to the host organism. ${ }^{1,2}$ Expression of these antigens on the cell surface requires the concerted action of multiple proteins, which are part of the antigen processing machinery, including the transporters associated with antigen processing (TAP) 1 and 2, the low molecular mass polypeptide (LMP), tapasin, and so on, ${ }^{3,4}$ which process the class 1 antigens in the endoplasmic reticulum (ER), after which the correctly folded heavy chain (class I molecules themselves) associates with the light chain ( $\beta 2 \mathrm{M}$ molecules) and are together transported to the cell surface. ${ }^{5}$ The proper expression thus results in the eventual presentation of the self/foreign peptides at the cell periphery, facilitating T-lymphocyte recognition.

Correlation between levels of MHC class I expression and pathological conditions have been demonstrated. Hypoexpression of class I molecules on the cell surface have been observed in many tumor cells due to various mechanisms including methylation of the heavy chain genes, defects in $\beta 2 \mathrm{M}$ expression, defects in TAP or LMP expression, and so on, hence leading to their escape from immune surveillance by the cytotoxic T cells. ${ }^{6}$ Reintroduction of the missing $\mathrm{MHC}$ class I genes in many mouse tumor models resulted in the susceptibility of tumor cells to immune destruction, ${ }^{7}$ demonstrating a crucial role for the expression of $\mathrm{MHC}$ class I genes in enabling the recognition by $\mathrm{T}$ cells. On the contrary, elevated expression of the MHC class I antigens leads to recognition and destruction by the immune $\mathrm{T}$ cells, as demonstrated in autoimmune diseases. ${ }^{8}$ Transgenic overexpression of class I genes resulted in cellular defects such as developmental failure in chimeras; induction of insulindependent diabetes due to destruction of pancreatic $\beta$-cells; thyroid cell destruction; neural tube defects; disruption of lens development leading to cataract induction, and demyelination in oligodendrocytes. ${ }^{9-13}$ Although many of the phenotypes observed were dependent on activating the immune system, some were independent of immune activation and often resulted in tissue destruction, suggesting that $\mathrm{MHC}$ class I expression may have other non-immunological functions regulating cellular growth. However, whether changes in class I expression levels affect the proper functioning of a cell (independent on the immune system) has not been critically analyzed.

We therefore tested the hypothesis that modulation of class I expression could directly affect cellular survival independent of the immune system. Our data indicate that cells lacking class I expression on the cell surface were resistant to a variety of apoptotic insults and had compromised p53 activation. Defective p53 was due to its sequestration in the cytoplasm. Cells lacking class I expression on the cell surface had elevated ER stress, which led to cytoplasmic sequestration of $\mathrm{p53}$. These data therefore suggest that defects in $\mathrm{MHC}$

\footnotetext{
${ }^{1}$ Laboratory of Molecular Carcinogenesis, Division of Cellular and Molecular Research, Humphrey Oei Institute of Cancer Research, National Cancer Centre Singapore, 11 Hospital Drive, Singapore, Singapore; ${ }^{2}$ Department of Biochemistry; National University of Singapore, 8 Medical Drive, Singapore, Singapore and ${ }^{3}$ Virtual Research Institute of Aging, Nippon Boehringer Ingelheim, Japan

*Corresponding author: K Sabapathy, Laboratory of Molecular Carcinogenesis, Humphrey Oei Institute of Cancer Research, National Cancer Centre, 11 Hospital Drive, Singapore 169610, Singapore.

Tel: + 656436 8349; Fax: + 656226 5694; E-mail: cmrksb@nccs.com.sg

Keywords: apoptosis; MHC class I; $\beta 2$ microglobulin; ER stress; p53; stress signals

Abbreviations: ATM, ataxia telangiectasia mutated; ER, endoplasmic reticulum; GSK, glycogen-synthase kinase; MHC, major histocompatibility complex; TAP, transporter-associated with antigen processing; TM, tunicamycin; TG, thapsigargin

Received 18.7.07; revised 01.2.08; accepted 20.3.08; Edited by V De Laurenzi; published online 30.5.08
} 
class I expression in tumors may provide not only escape from immune surveillance, but also confer survival advantage on tumor cells through the elevation of ER stress.

\section{Results}

$\beta 2 \mathrm{M}^{-I-}$ cells are resistant to cell death induced by genotoxic stress signals. Although sporadic reports have demonstrated that expression of $\mathrm{MHC}$ class I levels fluctuate during various physiological conditions, the relevance of such findings has not been fully understood. Hence, we first evaluated whether the absence of class I expression would affect cell death. As there are many class I antigens expressed in the cell, it is almost impossible to obtain cells devoid of all their expression. We therefore utilized primary mouse fibroblasts lacking $\beta 2 \mathrm{M}$, in which surface expression of $\mathrm{MHC}$ class I antigens have been almost completely lost (Figure 1a). ${ }^{14} \beta 2 M^{-1-}$ and $\beta 2 M^{+/+}$(wild type) primary embryonic fibroblast cells were derived from C57BL6/129 mouse embryos and tested for their sensitivity to nutrient deprivation and hypoxia, conditions prevalent in tumor masses in vivo. Cobalt chloride was used to induce hypoxia. Whereas wild-type cells were susceptible to apoptosis in both cases, as determined by annexin-V FITC and propidium iodide $(\mathrm{PI})$ staining, $\beta 2 \mathrm{M}^{-/}$cells were consistently more resistant (percentage viable cells - wild-type versus $\beta 2 \mathrm{M}^{-1-}$ cells $\rightarrow$ untreated: 96 versus $97 ; 0.1 \%$ serum: 55 versus $77 ; 400 \mu \mathrm{M} \mathrm{CoCl}_{2}: 43$ versus $67 ; 800 \mu \mathrm{M} \mathrm{CoCl}_{2}: 7$ versus 28) (Figure $1 \mathrm{~b}$, see bar chart for quantification), suggesting that the absence of $\beta 2 M$ (and hence $\mathrm{MHC}$ class I expression) may confer survival advantage to cells. We therefore tested whether $\beta 2 \mathrm{M}^{-1}$ cells would be resistant to other forms of genotoxic stress signals. Treatment of wildtype fibroblasts with cytotoxic drugs such as cisplatin and vinblastine or UV irradiation invariably led to cell death, which was markedly reduced in $\beta 2 M^{-1}$ cells (percentage viable cells - wild-type versus $\beta 2 \mathrm{M}^{-1-}$ cells $\rightarrow$ untreated: 88 versus 89; vinblastine: 58 versus 78; cisplatin: 7 versus 52; UV: 33 versus 70) (Figure 1c, lower panel for quantification). Similar results were also obtained with other cytotoxic agents such as doxorubicin, camptothecin, hydrogen peroxide, and $\gamma$-irradiation, determined using similar methods and also by independent analysis of sub-G ${ }_{1}$ DNA content (data not shown), indicating that expression of $\beta 2 \mathrm{M}$ is essential for optimal cell death.

To evaluate whether the surface expression of MHC class I antigens was required for optimal cell death, we utilized the pan $\mathrm{MHC}$ blocking antibody, W6/32, which has the ability to block surface expression of $\mathrm{MHC}$ class I antigens by virtue of recognition of a non-polymorphic epitope common to all HLA class I molecules. ${ }^{15}$ Human primary fibroblastic cell line IMR90 was incubated with W6/32 antibody before UV irradiation. Incubation with $\mathrm{W} 6 / 32$ resulted in the loss of surface expression of the human $\mathrm{MHC}$ class I antigens, as detected with another pan HLA antibody (Figure 1d, leftmost upper panel). The UV irradiation resulted in cell death to similar extents in cells incubated with control or W6/32 antibodies (percentage dead cells: - versus $+\mathrm{W} 6 / 32 \rightarrow$ 50J UV: 23 versus 21; 150J: 33 versus 31) (Figure 1d), indicating the blocking $\mathrm{MHC}$ class I expression on the cellular surface (as opposed to inappropriate expression of $\mathrm{MHC}$ b

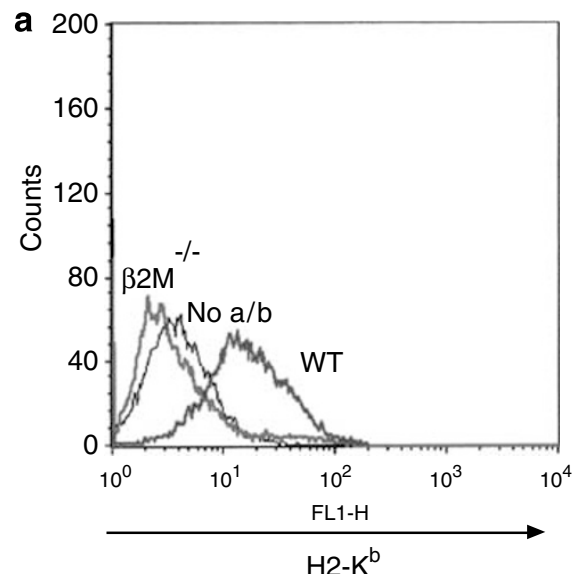

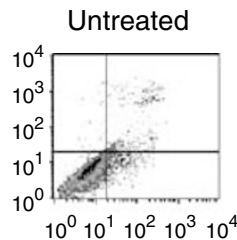
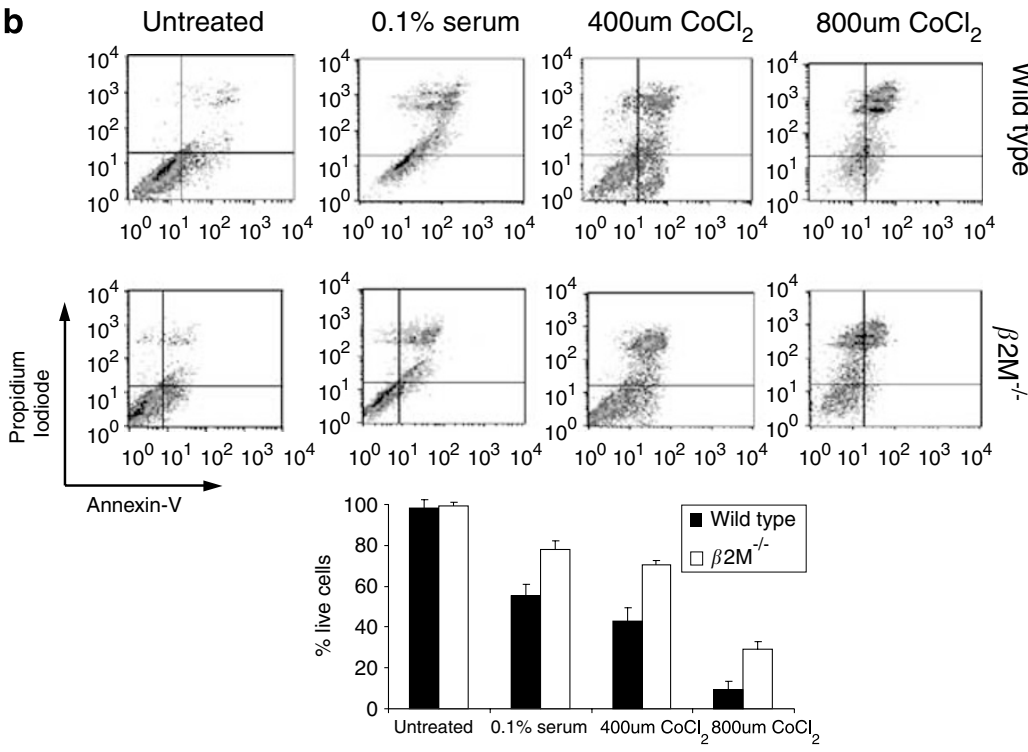

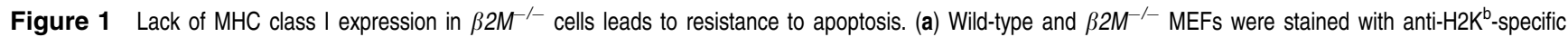
antibodies as indicated to determine class I expression on the cell surface by flow cytometric analysis. (b) Cells were starved off nutrients and cultured in $0.1 \%$ serum for $48 \mathrm{~h}$ or treated with cobalt chloride $\left(\mathrm{CoCl}_{2}\right)$ for $24 \mathrm{~h}$ to induce hypoxia, and the extent of cell death was determined by staining with annexin-V-FITC and propidium iodide (PI). Lower panel shows percentage live cells. All experiments were performed at least thrice and S.D. is indicated. (c) Cells were treated similarly with vinblastine (15 $\mu \mathrm{M})$, cisplatin $(50 \mu \mathrm{M})$, or UV irradiation $\left(40 \mathrm{~J} / \mathrm{m}^{2}\right)$ for $24 \mathrm{~h}$, and cell death was determined. Lower panel shows percentage live cells. (d) Human fibroblasts (IMR90 cells) were incubated without (-) or with ( + ) the anti-HLA antibody (W6/32) to block surface expression of MHC class I antigens. Cells were then stained with a different anti-HLA-A, B, C antibody (clone G46-2.6) to determine the extent of inhibition of expression of HLA antigens (top, left-most panel). Cells treated without or with W6/32 antibody were then UV irradiated and the extent of cell death was determined after $24 \mathrm{~h}$. Percentage dead cells are indicated 

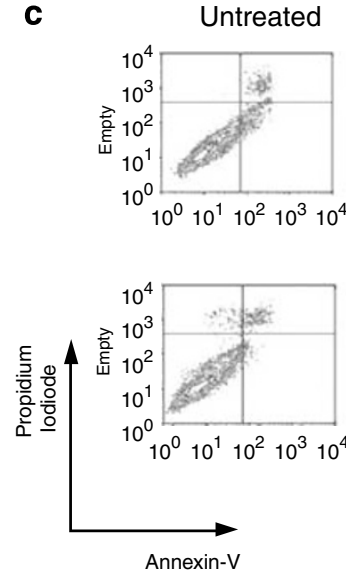

d
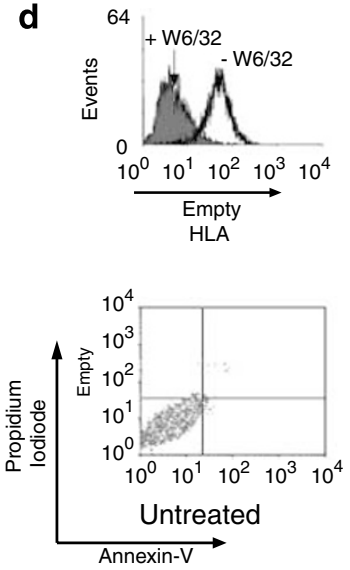

Vinblastine
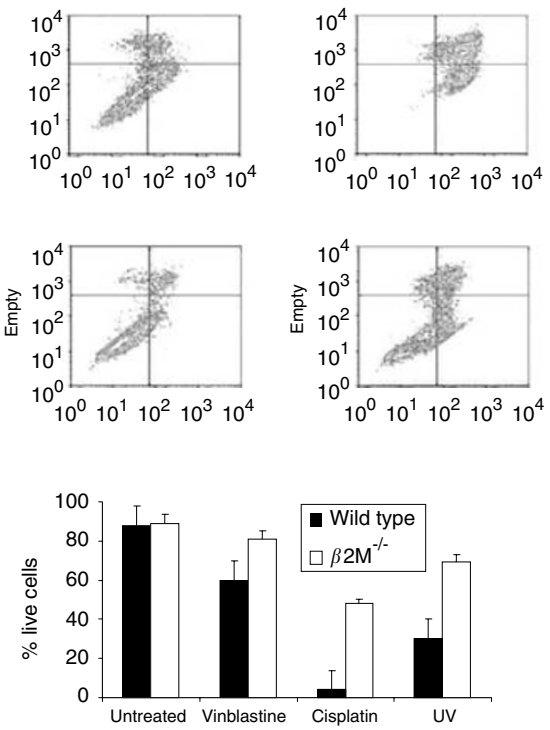
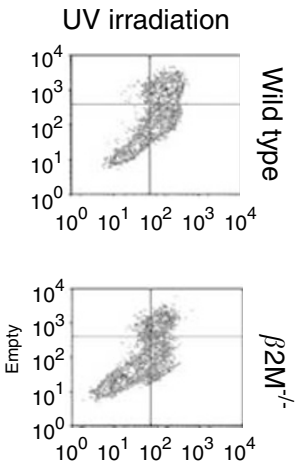

Figure 1 Continued.
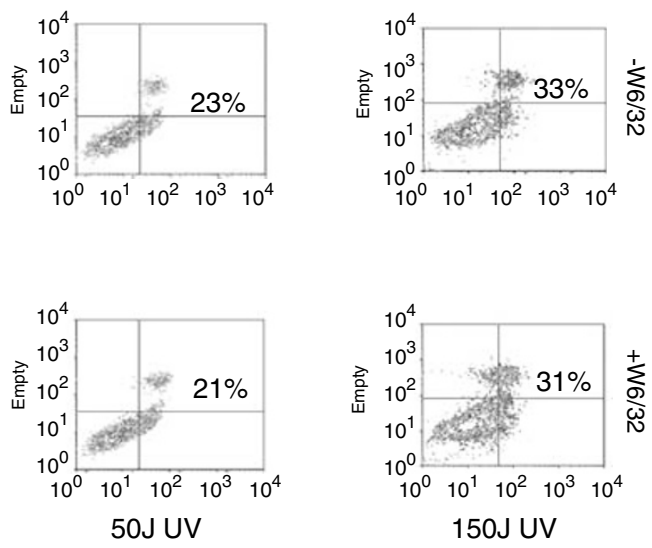

class I) does not affect sensitivity to cell death. Similar results were obtained with other genotoxic stresses (data not shown). Thus, the data together indicate that the absence of $\beta 2 \mathrm{M}$ affects the apoptotic process through mechanisms independent of surface exposure of the MHC class I antigens.

p53 activation is affected in $\boldsymbol{\beta 2} \boldsymbol{M}^{-1-}$ cells. p53 is a major regulator of cellular death, and is often functionally inactivated in tumors. ${ }^{16}$ Thus, we envisaged that loss of $\mathrm{MHC}$ class I expression leading to p53 inactivation could be one mechanism through which tumor cells acquire resistance to death, and hence, survive. Therefore, we next investigated whether $\beta 2 \mathrm{M}^{-1-}$ cells, which are resistant to cell death have compromised p53 activity. Immunoblot analysis indicated that whereas p53 protein was induced by various stress signals in wild-type mouse primary fibroblasts, it was severely compromised in $\beta 2 \mathrm{M}^{-1}$ cells (Figure $2 \mathrm{a}$, left panel). Time course analysis after $80 \mathrm{~J} / \mathrm{m}^{2}$ UV irradiation indicated that p53 was induced much later in the absence of $\beta 2 \mathrm{M}$ (Figure 1a, right panel), with delayed kinetics. Analysis of $p 53$ mRNA levels indicated that it was not affected by the absence of $\beta 2 \mathrm{M}$ in fibroblasts as well as other tissues (Figure 2b). Examination of the half-life of p53, by treatment with the protein synthesis inhibitor cyclohexamide and subsequent pulse chase, indicated that lack of $\beta 2 \mathrm{M}$ did not lead to reduced-p53 stability (Figure $2 c$ ).

We therefore examined whether p53 activation was affected in the absence of $\beta 2 \mathrm{M}$. Similar to earlier results, lower doses of UV irradiation $\left(20 \mathrm{~J} / \mathrm{m}^{2}\right)$ also resulted in the delayed accumulation of p53 protein in $\beta 2 M^{-}$cells (Figure 2d). While p53 accumulated at $1-2 \mathrm{~h}$ and reached the peak levels at 4-8 $\mathrm{h}$ post irradiation in wild-type cells, p53 was only visible at $4 \mathrm{~h}$ and reached peak levels at $24 \mathrm{~h}$ post irradiation in $\beta 2 \mathrm{M}^{-1}$ cells (Figure $2 \mathrm{~d}$; compare to loading controls and ratio of p53 to actin/tubulin). Analysis of posttranslation modifications such as phosphorylation at serines 18 and 389 (murine equivalent of serine 15 and 392, respectively) indicated that both these modifications were also delayed and compromised in $\beta 2 \mathrm{M}^{-/}$cells (Figure $2 \mathrm{~d}$ ). However, phosphorylation of ataxia telangiectasia mutated 

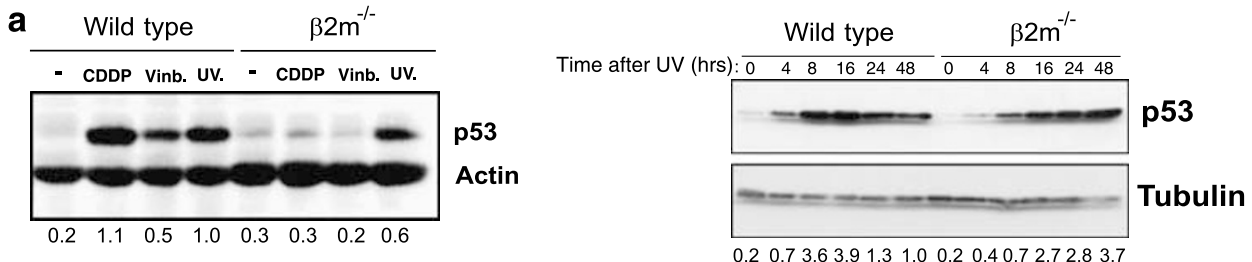

b

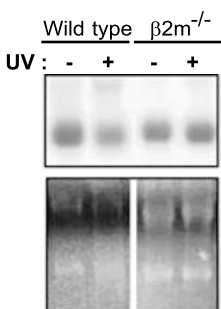

$p 53$

Loading control

Northern blot c

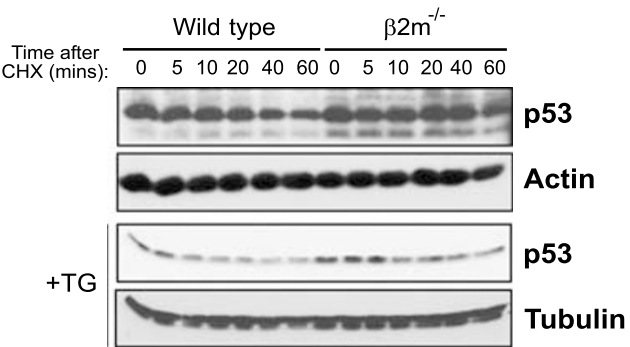

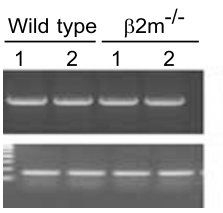

liver

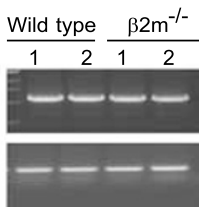

spleen

p53

tubulin

\section{RT-PCR}

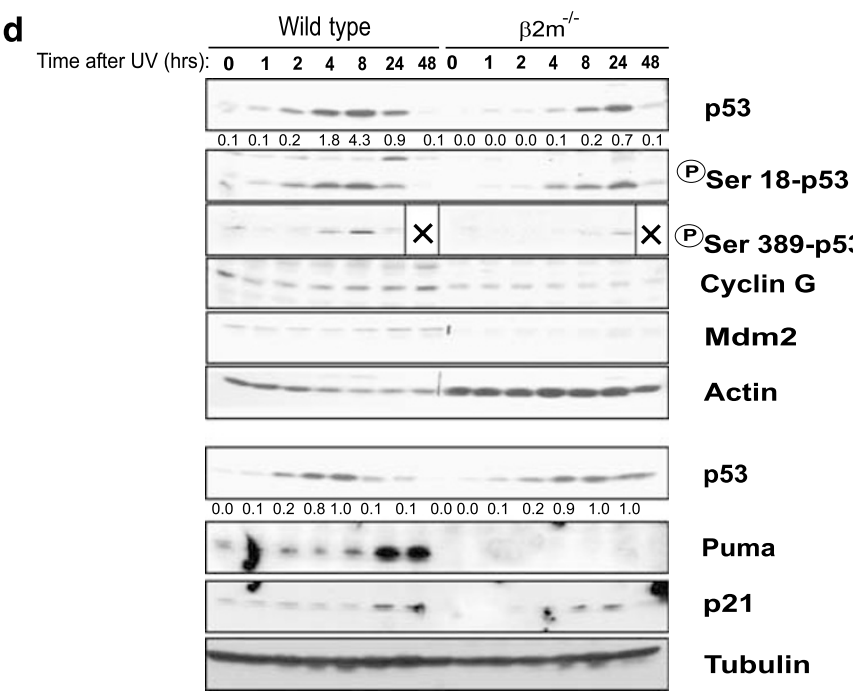

Figure 2 Lack of MHC class I expression in $\beta 2 M^{-1}$ cells affects p53 accumulation and activation. (a) Wild-type and $\beta 2 M^{-1-}$ MEFs were treated with various agents as indicated above (left panel) for $20 \mathrm{~h}$ or UV irradiated and cultured over time (right panel), and the amount of p53 was determined by immunoblotting with anti-p53 (CM5) antibody. Actin was used for loading control. CDDP: cisplatin. UV: $80 \mathrm{~J} / \mathrm{m}^{2}$. The ratio of p53 to actin or tubulin is indicated below the blots, rounded off to the nearest single decimal place. (b) The levels of p53 mRNA was determined as indicated upon UV irradiation by northern blotting (top panel). Liver and spleen tissues from wild-type and $\beta 2 M^{-1}$ mice were analyzed for $553 \mathrm{mRNA}$ expression by reverse transcriptase-PCR (RT-PCR) reaction (bottom panel). Two samples from different mice were used in each case (1 and 2). (c) p53 levels were determined by immunoblotting in wild-type and $\beta 2 M^{-1-}$ MEFs treated without or with the ER stress inducer thapsigargin (TG) and subsequently with the protein synthesis inhibitor cyclohexamide (CHX, $25 \mu \mathrm{g} / \mathrm{ml})$ for the indicated time periods. (d) Time course analysis of steady state levels of total p53, phosphorylated p53 (ser18 and 389), and p53 target genes such as Puma, Cyclin G, Mdm2, and p21 were analyzed after UV irradiation (20 J/m²). The ratio of p53 to actin or tubulin is indicated below the p53 blots

(ATM) protein at serine 1981, a critical event for its activation and the eventual phosphorylation of mouse p53 on serine 18 , and its activation was not affected after $\gamma$-irradiation in $\beta 2 \mathrm{M}^{-1-}$ cells (data not shown). Consistent with the compromised activation of $p 53$, the induction of $p 53$ target genes such as PUMA, Cyclin G, MDM2, and p21 was reduced in $\beta 2 M^{-1}$ cells (Figure $2 d$ ). Taken together, the data indicate that lack of $\beta 2 \mathrm{M}$ results in delayed and compromised activation of p53. 

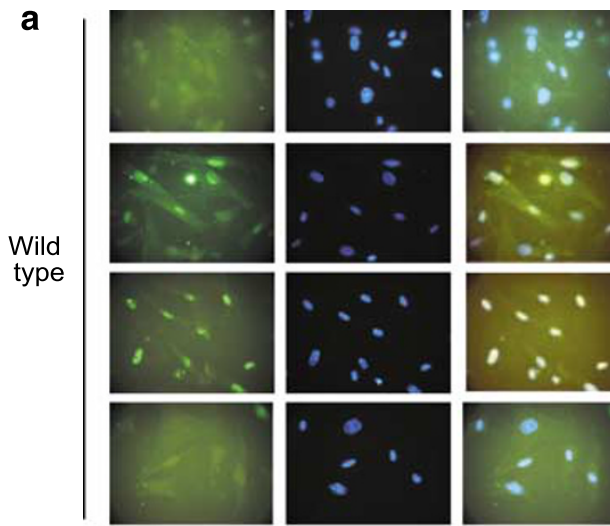

Untreated
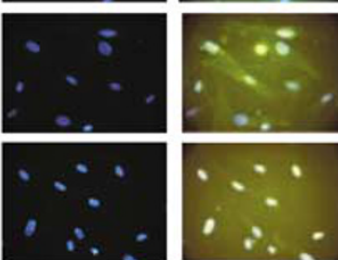

UV irradiated,

$6 \mathrm{hrs}$

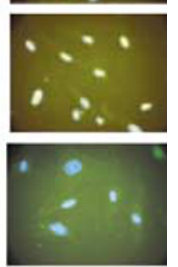

UV irradiated

$18 \mathrm{hrs}$
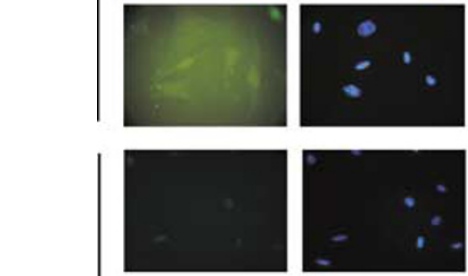

Tunicamycin treated, $18 \mathrm{hrs}$
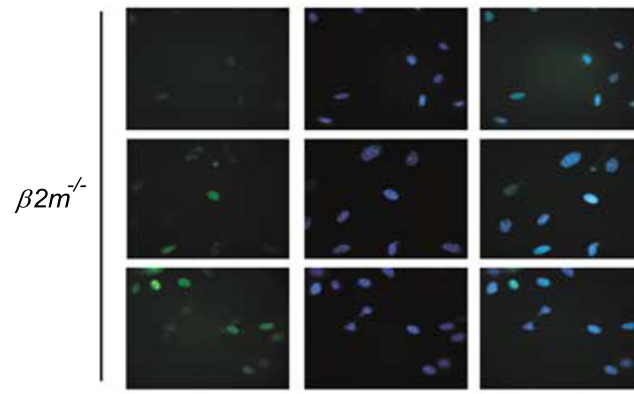

Untreated
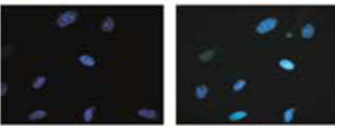

UV irradiated,

6hrs
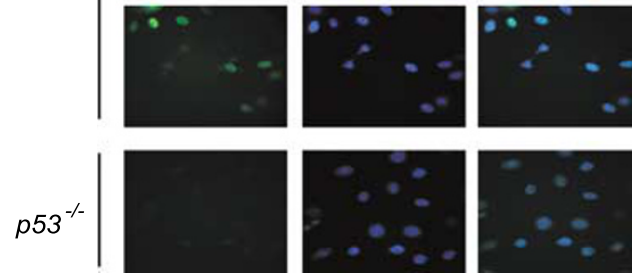

UV irradiated, $18 \mathrm{hrs}$

p53
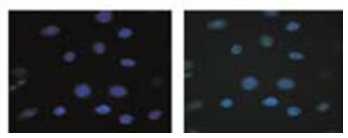

Untreated

dapi

merge

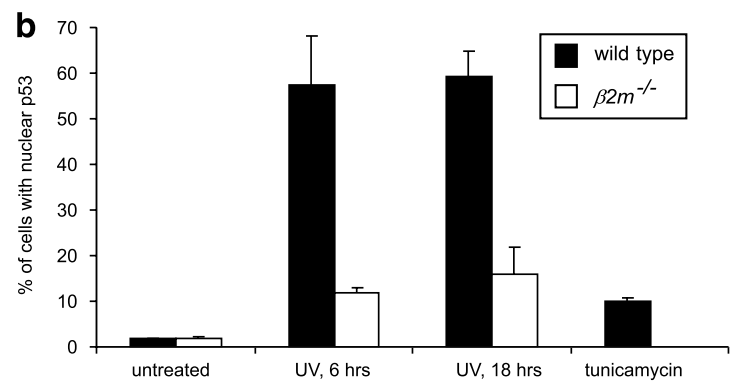

C

Wild type

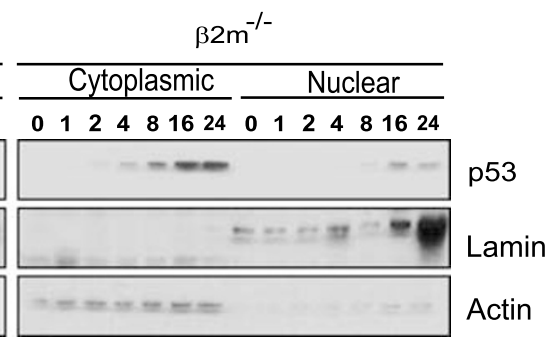

Figure 3 Defects in cytoplasmic translocation of $\mathrm{p} 53$ upon UV irradiation in $\beta 2 \mathrm{M}^{-1-}$ cells. (a and $\mathbf{b}$ ) Wild-type and $\beta 2 \mathrm{M}^{-1-}$ MEFs were UV irradiated ( $40 \mathrm{~J} / \mathrm{m}^{2}$ ) for 6 or $18 \mathrm{~h}$ or treated with the endoplasmic stress inducer tunicamycin $(10 \mu \mathrm{g} / \mathrm{ml})$ for $18 \mathrm{~h}$ and the cells were stained with anti-p53-specific antibody or dapi to locate the nucleus. Immunofluorescence analysis was performed to determine the extent of p53 translocation into the nucleus upon UV irradiation (a). Tunicamycin treatment has been shown to result in p53 being present in the cytoplasm (and not translocate to the nucleus) and was used as a control. Between 150 and 200 cells were counted per field, and at least three independent fields were analyzed per experiment. The experiments were repeated at least four independent times. (b) Quantification of cells (average) with nuclear p53 out of the whole field of view from one representative experiment. (c) Both nuclear and cytoplasmic fractions were prepared from both cell types after UV irradiation for the indicated time periods and the levels of p53 was determined. Lamin and actin served as quality and loading controls for nuclear and cytoplamsic fractions, respectively 
p53 nuclear translocation is affected in $\beta 2 \mathbf{M}^{-1-}$ cells. One mechanism by which p53 activation can be compromised is due to the cytoplasmic sequestration of $p 53$, as has been demonstrated in neuroblastoma cells and in cells undergoing endoplasmic reticulum stress. ${ }^{17,18}$ Hence, we evaluated whether cytoplasmic nuclear localization of p53 is affected in $\beta 2 M^{-1-}$ cells. To this end, both wild-type and $\beta 2 \mathrm{M}^{-1}$ cells were UV irradiated and the location of endogenous p53 was monitored by immuno fluorescence after staining with p53-specific antibodies. p53, which was generally diffuse and almost undetectable in untreated wildtype cells was localized to the nucleus, starting at about $6 \mathrm{~h}$ and peaked at around $18 \mathrm{~h}$ post irradiation (Figure 3a, top panel). Whereas only $1.75 \%$ of the cells had p53 in the nucleus in untreated state, irradiation resulted in about 57$59 \%$ of the wild-type cell population containing nuclear p53 (Figure 3b). Consistent with published findings, treatment of wild-type cells with tunicamycin (TM), an ER stress-inducing agent, did not result in nuclear accumulation of p53 (Figure $3 a$, top panel). ${ }^{18}$ However, irradiation of $\beta 2 M^{-1-}$ cells did not result in substantial nuclear accumulation of p53 at both the time points, leading only to about $12-16 \%$ of the $\beta 2 \mathrm{M}^{-1-}$ cells containing nuclear p53 (Figure 3a, middle panel). These results thus indicate that p53 localization to the nucleus might be affected in $\beta 2 \mathrm{M}^{-1-}$ cells, which could contribute to the defects in p53 activation in these cells.

To confirm this, we performed fractionation of cellular lysates and analyzed the p53 status in the cytoplasmic and nuclear fractions. As shown in Figure 3c, UV irradiation resulted in an increase in p53 levels in cytoplasm from 2-8 h and a consequent accumulation in the nucleus between 4$16 \mathrm{~h}$ in wild-type cells. By contrast, although p53 accumulated later in $\beta 2 \mathrm{M}^{-1-}$ cells (between 16-24 h), there was a distinct absence of accumulation in the nuclear fraction (Figure $3 c$, compare with loading controls). These data, together with the immunofluorescence results suggest that p53 translocation to the nucleus upon exposure to genotoxic stress such as UV irradiation is affected in the absence of $\beta 2 \mathrm{M}$.

$\beta 2 M^{-1-}$ cells display constitutively elevated ER stress. Sequestration of p53 in the cytoplasm could be due to several mechanisms, one of which is elevated ER stress, as has been recently demonstrated. ${ }^{18}$ As the absence of $\beta 2 \mathrm{M}$ results in the improper folding of the $\mathrm{MHC}$ class I antigens (leading to defects in surface expression), we envisaged that this would lead to ER stress in these cells. We therefore tested whether the $\beta 2 \mathrm{M}^{-/}$cells were subjected to constitutive ER stress. To this end, we employed several assays to determine the ER stress status. Firstly, the status of $G R P 78$, which is activated by ER stress ${ }^{19}$ was evaluated. Untreated wild-type cells did not express GRP78, which was induced by treatment with TM or another ER inducer, thapsigargin (TG) (Figure 4a). By contrast, GRP78 expression was already detected in untreated $\beta 2 \mathrm{M}^{-1-}$ cells, which were further robustly induced upon TM or TG treatment (Figure 4a), suggesting that the $\beta 2 \mathrm{M}^{-1-}$ cells are subjected to ER stress even in the absence of drug treatment. Next, we determined the status of $X B P-1$, which is also induced by ER stress. ${ }^{20}$ Similar to GRP78, untreated $\beta 2 \mathrm{M}^{-/}$cells expressed significantly higher basal levels of
$X B P-1$ compared to their wild-type counterparts (Figure $4 \mathrm{~b}$, top panel). In both cell types, $X B P-1$ expression was induced upon treatment with TG or TM. $X B P-1$ is generally found in an unprocessed state and is processed by splicing of a small intron upon ER stress, giving rise to bands of different sizes upon Pst 1 restriction enzyme digestion. ${ }^{21}$ Analysis upon Pst1 digestion also revealed elevated levels of processed product from untreated $\beta 2 \mathrm{M}^{-1}$ cells compared to wild-type cells (Figure 4b, lower panel).

ER stress has also been shown to result in the phosphorylation and activation of the ER-resident kinase, PERK. ${ }^{22}$ Analysis of phosphorylation status of PERK indicated that it was constitutively and robustly phosphorylated in $\beta 2 \mathrm{M}^{-1-}$ cells compared to wild-type cells (Figure 4c). The levels of total PERK was not affected, and treatment with TM or TG resulted in phosphorylation of PERK in wild-type cells, confirming its role in ER stress activation (Figure 4c).

As ER stress was shown to inactivate p53 function due to the physical interaction with GSK-3 $\beta$, leading to sequestration of $\mathrm{p} 53$ in the cytoplasm, ${ }^{18}$ we tested whether p53 was constitutively bound to GSK-3 $\beta$ in $\beta 2 \mathrm{M}^{-/}$cells. Immunoprecipitation with anti-p53 antibodies and subsequent immunoblot with anti-GSK-3 $\beta$ antibodies indicated that GSK-3 $\beta$ was only bound to p53 upon ER stress induced by TG in wild-type cells (Figure $4 d$ ). However, GSK-3 $\beta$ was found to be bound to p53 in the untreated state in $\beta 2 \mathrm{M}^{-/-}$cells (Figure $4 \mathrm{~d}$ ), indicative of elevated ER stress in these cells. The total levels of GSK-3 $\beta$ were equal in both cell types, thereby excluding any alterations in GSK-3 $\beta$ levels in $\beta 2 \mathrm{M}^{-1}$ cells that may have contributed to the observations (Figure $4 d$, lower panel).

To further confirm these observations, we performed immunofluorescence studies to evaluate the localization of GSK-3 $\beta$, which was shown to be enriched upon ER stress. ${ }^{18}$ Consistently, we found that GSK-3 $\beta$ was diffused in untreated cells and was enriched in the nucleus upon TG treatment in wild-type cells (Figure 4e, left panel). However, GSK-3 $\beta$ was found both in the nucleus and the cytoplasm of untreated $\beta 2 \mathrm{M}^{-/}$cells to a higher extent (Figure $4 \mathrm{e}$, right panel). To substantiate this observation, we utilized nuclear and cytoplasmic fractions from cells of both genotypes and found that GSK-3 $\beta$ levels were indeed elevated in the nuclear fraction of $\beta 2 M^{-1-}$ cells compared to wild-type cells (Figure 4f; compared with loading control). As expected, more p53 was seen in the nuclear fraction of wild-types cells in contrast to $\beta 2 \mathrm{M}^{-1}$ cells after UV irradiation (Figure 4f). Thus, the data together suggest that the absence of $\beta 2 \mathrm{M}$ results in constitutively elevated ER stress, thereby probably leading to the sequestration of $p 53$ in the cytoplasm.

Loss of MHC class I expression due to TAP deficiency also results in defects in apoptosis, p53 activation and elevated ER stress. To evaluate whether the lack of class I expression due to other defects in the MHC processing pathway would also result in a similar phenotype, we employed the mouse RMA and RMAS isogenic tumor cells that are proficient or deficient in TAP expression, respectively. ${ }^{23}$ TAP has been demonstrated to be required for the efficient expression of MHC class I on the cellular surface. ${ }^{24}$ Flow cytometric analysis confirmed that RMAS cells have a significant reduction of surface expression of the 
$\mathrm{H}-2 \mathrm{k}^{\mathrm{b}}$ antigen (Figure 5a). Cell death analysis indicated that whereas RMA cells were sensitive to a variety of cellular stress insults such as cisplatin, $\gamma$ - and UV irradiation, RMAS cells were significantly more resistant to cell death (percentage dead cells $\rightarrow$ RMA versus RMAS - untreated: 2.5 versus 3.5 ; cisplatin: 52.7 versus 24.3 ; IR: 38.5 versus 23.5; UV: 67.1 versus 29.2) (Figure $5 b$ ), similar to the observation with $\beta 2 \mathrm{M}^{-1}$ cells. Moreover, p53 levels after a

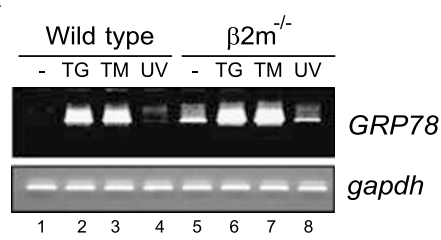

b

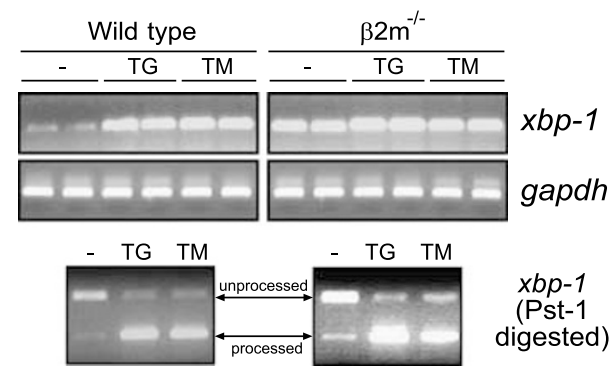

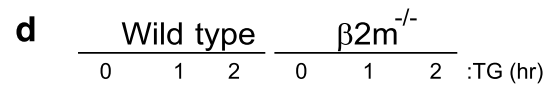

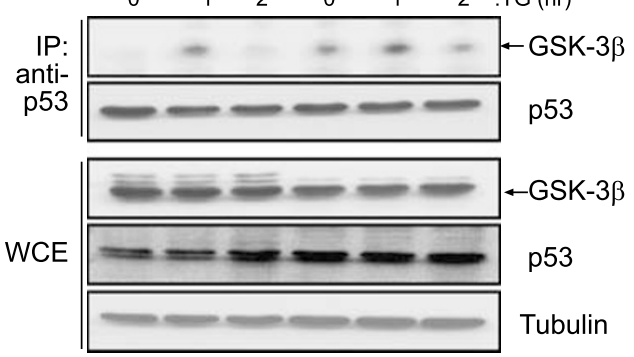

$\beta 2 \mathrm{~m}^{-/-}$

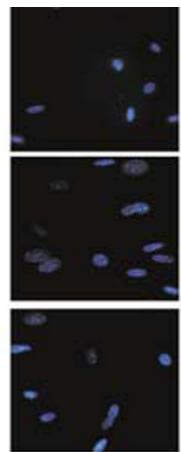

dapi

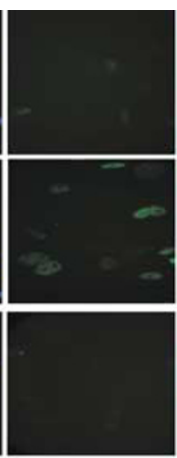

p53

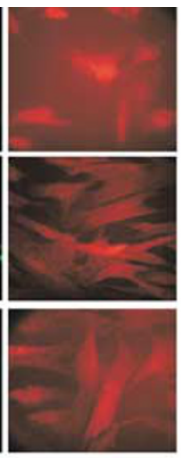

GSK-3 $\beta$

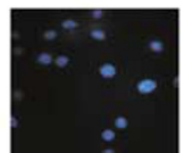

dapi

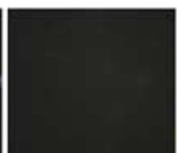

secondaryFITC alone

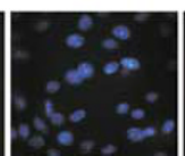

dapi Untreated UV irradiated

TG treated

secondary-

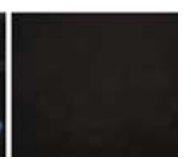
$\mathrm{PE}$ alone

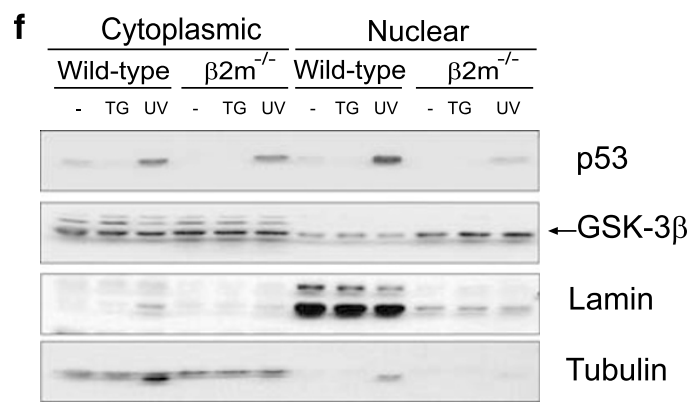



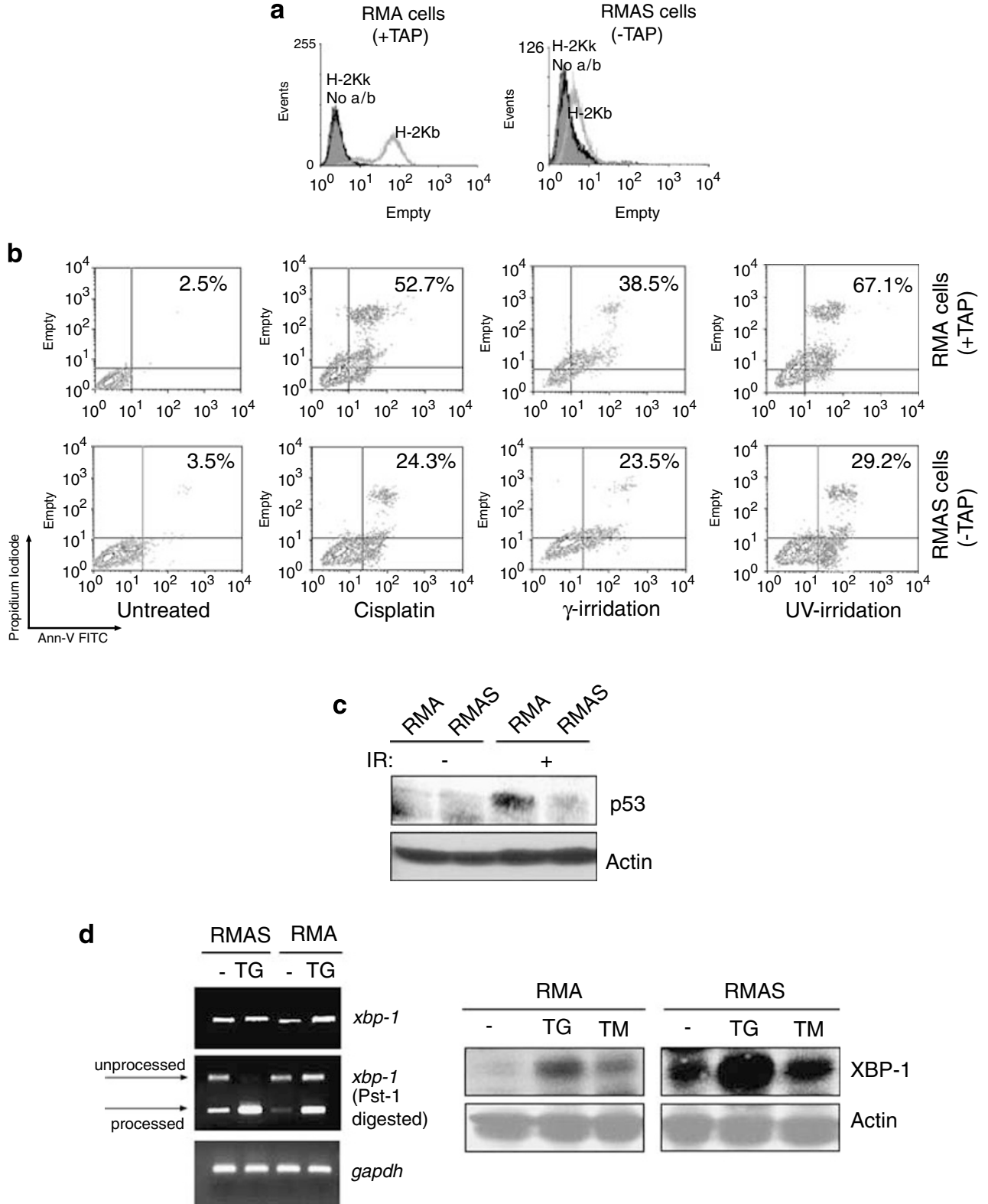

Figure 5 Lack of MHC class I expression in TAP-deficient RMAS cells also lead to enhanced resistance to apoptosis and elevated ER stress. (a) Transporter associated with antigen processing (TAP) proficient (RMA) and deficient (RMAS) isogenic cells were stained with anti-H-2k ${ }^{b}$ and $\mathrm{H}-2 \mathrm{k}^{\mathrm{k}}$ antibodies. RMAS cells have markedly reduced expression of $\mathrm{H}-2 \mathrm{k}^{\mathrm{b}}$. (b) RMA and RMAS cells were treated with cisplatin $(50 \mu \mathrm{M})$ or UV $\left(50 \mathrm{~J} / \mathrm{m}^{2}\right)$ or $\gamma$ - irradiated $(20$ Gy), and cell death was determined after $20 \mathrm{~h}$, as described. Percentage dead cells are indicated. (c) Immunoblot analysis of p53 levels after $\gamma$-irradiation (20 Gy, $16 \mathrm{~h}$ ) of RMA and RMAS cells is shown. (d) Reverse transcriptase-PCR (RT-PCR; left panel) and immunoblot (right panel) analysis of XBP-1 was performed using RMA and RMAS cells, as described above

Figure $4 \quad \mathrm{~B}_{2 \mathrm{M}^{-1}}$ cells lacking MHC class I expression display constitutively elevated endoplasmic stress response. (a) Reverse transcriptase-PCR (RT-PCR) analysis of GRP78 was performed using mRNA from untreated or cells treated with thapsigargin (TG; $1 \mu \mathrm{M})$, tunicamycin (TM; $10 \mu \mathrm{g} / \mathrm{ml})$, or UV irradiation (UV; $40 \mathrm{Jm}^{-2}$ ) for $6 \mathrm{~h}$. Gapdh serves as loading control. (b) RT-PCR analysis was performed to evaluate the levels of XBP-1, as described above (upper panel). Duplicate samples were used from each set. The XBP-1 PCR product was isolated and digested with Pst-1 restriction enzyme, which distinguishes between the processed ( $300 \mathrm{bp}$ ) and unprocessed band (600 bp; lower panel). XBP-1 is processed upon endoplasmic reticulum stress. (c) Immunoblot analysis of phosphorylated or total PERK in these cells. (d) Whole cell extracts (WCE) from both wild-type and $\beta 2 M^{-1-} \mathrm{MEFs}$, which were treated with TG for the indicated time periods were used for immunoprecipitation with anti-p53 antibodies, and immunoblotted

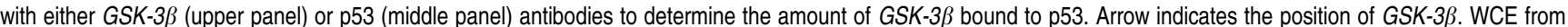
above cells were immunoblotted with anti-GSK-3 $\beta$ and p53 antibodies to determine total the amount of protein present (lower panel). (e) Immunofluorescence analysis was performed using untreated or cells treated with UV and TG to determine the localization of GSK-3 $\beta$ and p53, as described above. Dapi staining shows the position of nuclei.

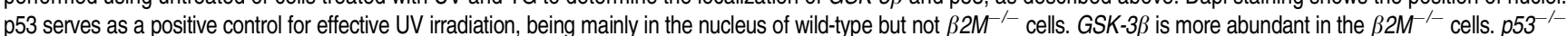
cells were used as a negative control for the background staining with the p53 antibody. Additionally, cells were stained only with FITC or PE-conjugated secondary antibodies as controls. (f) Both nuclear and cytoplasmic fractions were prepared from both cell types after UV irradiation or TG treatment and the levels of p53 or GSK-3 $\beta$ were determined, as described above 
$\gamma$-irradiation was also compromised in RMAS cells compared to RMA cells (Figure $5 \mathrm{c}$ ). Analysis of ER stress markers such as $X B P-1$ levels indicated that untreated RMAS cells contained higher levels of XBP-1 compared to RMA cells (Figure 5d, left top panel). Moreover, Pst1 restriction digestion experiments indicated that RMAS cells contained significantly higher levels of processed products even in unstressed state (Figure $5 \mathrm{~d}$, left middle panel). In addition, western blot analysis confirmed that RMAS cells expressed higher XBP-1 protein even without any ER stress inducers (Figure $5 d$, right panel). Taken together, the data indicate that absence of $\mathrm{MHC}$ class I expression due to defects in various mechanisms result in elevated ER stress and consequently, defective p53 activation and resistance to apoptosis.

\section{Discussion}

The data presented here demonstrate that the defects in $\mathrm{MHC}$ class I surface expression result in elevated ER stress and defective p53 activation. This leads to resistance to multiple apoptotic stimuli, therefore conferring cellular survival advantage. These results thus identify a novel role for defects in $\mathrm{MHC}$ class I antigen expression in promoting cellular growth and survival by allowing escape from apoptotic pressure.

Although sporadic reports have demonstrated that expression of $\mathrm{MHC}$ class I levels fluctuate during various physiological conditions, the relevance of such findings has not been fully understood. The data presented here show that reduced class I expression confers survival advantage in response to growth factor deprivation, hypoxia, and a variety of genotoxic stresses. It is to be noted that oncogene-mediated cellular transformation and subsequent tumorigenesis - in which conditions of hypoxia and nutrient deprivation prevail - are also associated with reduction in class I expression. ${ }^{25-27}$ Conversely, treatment with genotoxic agents leading to cell cycle arrest was associated with increased class I expression. Supporting evidence comes from pathological conditions in which cells undergoing arrest or cell death have also been associated with increased MHC class I expression. For example, motoneurons and nigral dopaminergic neurons in the brainstem of adult rat which were vulnerable to neurodegeneration in diseases such as Parkinson's disease (PD) and amyotrophic lateral sclerosis display high levels of both $\mathrm{MHC}$ class I heavy chain and $\beta 2 M$ mRNAs. ${ }^{28}$ Moreover, MHC class I expression was elevated in microglia upon both ischemia and axonal degeneration. ${ }^{29}$ Similarly, we have noted that overexpression of $\mathrm{MHC}$ class I molecules also lead to p53-dependent apoptosis in fibroblasts (data not shown). Thus, these evidences from physiological settings correlate surface MHC class I levels with cellular survival, supporting our observations using cultured cells.

Defects in MHC class I expression were found to result in ER stress, which led to defects in p53 activation due to cytoplasmic sequestration of p53. p53 is a known mediator of cell death, and functional inactivation often occurs in tumors. ${ }^{16}$ Thus, loss of MHC class I expression leading to p53 inactivation may thus be one mechanism through which tumor cells acquire resistance to death, and hence survive. A recent report shows that downregulation of $\mathrm{MHC}$ class I expression correlated with poor prognosis in colon cancers is supporting this idea. ${ }^{30}$ Besides, several reports have suggested a positive correlation between elevated ER stress and resistance to apoptosis, and between an increase in ER markers and carcinogenesis. Overexpression of GRP78 was shown to result in reduced apoptosis and higher colony survival upon genotoxic challenges, and has been associated with hepatocarcinogenesis and malignant breast lesions, and very recently, to be a predictor of chemoresistance. ${ }^{31-34}$ Moreover, PERK function was shown to be essential for cell survival following the exposure of cells to ER stress. ${ }^{35}$ Furthermore, XBP-1 was also shown to be essential for survival under hypoxic conditions and is required for tumor growth. ${ }^{36}$ Finally, reduction of expression of ER proteins correlated with lower viability after ionophore treatment. ${ }^{37}$ Taken together, the evidence supports our proposal that defects in cell-surface $\mathrm{MHC}$ class I expression lead to resistance to apoptosis by elevating ER stress.

As p53 nuclear localization and consequently its activity is compromised due to lack of MHC class I expression, we also investigated whether lack of class I in $\beta 2 M^{-1}$ mice would affect tumorigenicity. However, $\beta 2 \mathrm{M}^{-1-}$ mice did not succumb to spontaneous tumors up to over 3 years unlike their $p 53^{-/-}$ counterparts (data not shown). This suggested that although p53 activation (such as apoptosis) is compromised in $\beta 2 \mathrm{M}^{-/-}$ cells, it was not sufficient to allow the formation of spontaneous tumors in mice. This could be due to the requirement for a threshold of p53 activity that has to be maintained for tumor suppression (which was not breached in the $\beta 2 \mathrm{M}^{-1}$ mice) implying that although p53 activity is compromised in $\beta 2 \mathrm{M}^{-1-}$ mice, it is not sufficient enough to inhibit p53's tumor suppressive function. In this respect, we noted that not all p53-dependent functions, such as ras-mediated senescence of primary fibroblasts as well as basal cellular proliferation rates, were compromised by lack of $\beta 2 M$ (data not shown). Thus, although the defects in MHC class I surface expression is not sufficient to promote tumorigenicity, its absence leading to resistance to apoptosis probably contributes to the tumorigenic process.

In conclusion, the data presented here suggest that defects in $\mathrm{MHC}$ class I expression often found in cancer cells may not only provide an avenue for escape from immune surveillance, but also directly result in their resistance to apoptotic stimuli, thereby allowing enhanced growth. Thus, the status of surface $\mathrm{MHC}$ class I levels in tumors may provide information on whether the tumors may respond well to cytotoxic drugs, and be of prognostic significance. In addition, future efforts aimed at identifying regulators of ER stress may have potential utility in treating class I deficient tumors.

\section{Materials and Methods}

Cell lines, transfections, treatment conditions, and plasmids. All cell lines used in this study were cultured in $10 \%$ fetal bovine serum containing DMEM. IMR90 human diploid fibroblasts were obtained from ATCC. RMA and RMAS cells were obtained from Dr. Hideo Yagita and grown as non-adherent cells in culture. Murine embryonic fibroblasts (MEFs) were isolated from E12.5 $\mathrm{p} 53^{-/-}$ and $\beta 2 \mathrm{M}^{-1}$ embryos and as described and immortalized spontaneously according to the $3 \mathrm{~T} 3$ protocol. ${ }^{38}$ Cells were starved by culturing in medium containing $0.5 \%$ serum for $48 \mathrm{~h}$, and subsequently, 20\% FCS (final concentration) was added directly onto the dishes to release them into the cell cycle. Nutrient deprivation was achieved 
by culturing cells $0.1 \%$ serum containing medium without other amino acids and nutrients.

Cells were treated with the following stress signals for cell death and MHC class I analysis: UV irradiation (as indicated); $\gamma$-irradiation (20 Gy); cobalt chloride (400 and $800 \mu \mathrm{M})$; vinblastine $(15-30 \mu \mathrm{M})$; cisplatin $(50-80 \mu \mathrm{M})$; TM $(10 \mu \mathrm{g} / \mathrm{ml})$, and TG $(1 \mu \mathrm{M})$, for indicated time periods. Cells were treated with $25 \mu \mathrm{g} / \mathrm{ml}$ cyclohexamide to inhibit protein synthesis for the indicated time periods.

Immunoblot analysis. Immunoblot analysis was performed as described. ${ }^{38}$ Cellular fractionation was performed using NE-PER nuclear and cytoplasmic extractions reagents (Pierce Biotech), according to the manufacturer's protocol. The following antibodies were used for immunoblot analysis: p53 (CM5 - Novocastra) and (IC12 - Cell Signaling), phospho-ser18, -ser 315, and -389 p53, GSK-3 $\beta$ (Cell Signaling), phospho-PERK (US Biologicals), MDM2, Puma, Cyclin G, XBP-1, PERK, p21, Tubulin (Santa Cruz), Lamin (Cell Signaling), and Actin (Sigma).

Immunoprecipitations were carried out using anti-p53 antibody (mixture of Ab-3 and Ab-4, Oncogene Sciences), and immunoblotted using anti-p53 (CM5 or IC12) or anti-GSK3 $\beta$ antibodies.

Flow cytometry and cell death assays. Flow cytometric analysis was performed to determine MHC class I surface expression, using either the anti-H-2K or $\mathrm{H}-2 \mathrm{~K}^{\mathrm{k}}$ (Pharmingen) antibodies. Cell-surface MHC class I expression was blocked by incubated with the anti-pan HLA-blocking antibody (W6/32) for $1 \mathrm{~h}$ before analysis of HLA surface expression using another anti-pan HLA antibody (clone G46-2.6, Pharmingen).

Two independent cell death assays were employed in this study, namely PI staining of sub-G1 populations, and annexin-V staining. Sub-G1 population analysis representing dead cells was performed as for cell cycle status analysis. To detect annexin- $\mathrm{V}^{+}$cells, cells were incubated with FITC-conjugated annexin-V (BD biosciences) and $50 \mu \mathrm{g} / \mathrm{ml} \mathrm{PI}$ in binding buffer, and cells were analyzed by flow cytometry.

RNA analysis. Total RNA was extracted and utilized for reverse transcriptasePCR (RT-PCR) analysis as described ${ }^{39}$ using the following primers: GRP78 - for: 5'- TTGCTGGACTCCTAAGACTC-3'; rev: 5' -AGCAGATCAGTGCACCTACA-3' (expected size: approximately $2.0 \mathrm{~Kb}$ ); XBP-1 - For: 5'- AAACAGAGTAGCA GCGCAGACTGC-3'; Rev: 5'-GGATCTCTAAAACTAGAGGCTTGGTG-3' (600 bp); p53 - For: 5'- GCAATTCGCCATGGAGGAGTCACAGTCG-3'; rev: 5'- CCCAAGC TTCAGTCTGAGTCAGGCCCCAC-3' (1.2 Kb); Tubulin - For: $5^{\prime}$ - CCACGTC AAGACGGCCGTGTG-3'; Rev: 5'- GACAGAGGCAAACTGAGCACC-3' (436 bp); gapdh - For: 5'- ATCTTCTTGTGCAGTGCCAG-3'; rev: 5'-GTAGTTGAGGTCA ATGAAGG-3' (140 bp). XBP-1 product was digested with Pst-1 restriction enzyme to determine the amount of processed or unprocessed products of 300 and $600 \mathrm{bp}$, respectively, as described. ${ }^{21}$

Northern blot was performed using mouse p53 cDNA as a probe as described. ${ }^{38}$

Immunofluorescence. Cells grown on cover slips were fixed in $4 \%$ paraformaldehyde/PBS and incubated with anti-p53 (mixture of Ab-3 and Ab-4), or anti-GSK3 $\beta$ antibodies, followed by incubation with the secondary antibody (FITC or PE-conjugated anti-mouse/rabbit $\operatorname{lgG}$ ) before being mounted in mounting medium containing DAPI. Cells were counted by eye, and compared to negative controls such as those without primary antibody as well as with $p 53^{-1-}$ cells stained with anti-p53 antibody. Cells with signals above these negative controls were counted as positive events. Between 150 and 200 cells were counted per field, and at least three independent fields were analyzed per experiment. The experiments were repeated at least four independent times.

Acknowledgements. We thank Tan YT for technical assistance during the early phase of the project, Dr. Hideo Yagita for the RMA/RMAS cells, and Dr. Hui KM for the W6/32 antibody. This work was supported by the generous funding by the National Medical Research Council of Singapore (NMRC) and Nippon Boehringer Ingelheim, Japan, to KS.

1. Janeway Jr CA. The role of self-recognition in receptor repertoire development. Members of the Janeway Laboratory. Immunol Res 1999; 19: 107-118.

2. David-Watine $B$, Israel $A$, Kourilsky $P$. The regulation and expression of MHC class I genes. Immunol Today 1990; 11: 286-292.

3. Elliott T, Williams A. The optimization of peptide cargo bound to MHC class I molecules by the peptide-loading complex. Immunol Rev 2005; 207: 89-99.
4. Groothuis TA, Griekspoor AC, Neijssen JJ, Herberts CA, Neefjes JJ. MHC class I alleles and their exploration of the antigen-processing machinery. Immunol Rev 2005; 207: 60-76.5.

5. Flutter B, Gao B. MHC class I antigen presentation - recently trimmed and well presented. Cell Mol Immunol 2004; 1: 22-30.

6. Garrido F, Algarra I. MHC antigens and tumor escape from immune surveillance. Adv Cancer Res 2001; 83: 117-158.

7. Hui KM. Re-expression of major histocompatibility complex (MHC) class I molecules on malignant tumor cells and its effect on host-tumor interaction. Bioessays 1989; 11: 22-26.

8. Singer DS, Mozes E, Kirshner S, Kohn LD. Role of MHC class I molecules in autoimmune disease. Crit Rev Immunol 1997; 17: 463-468.

9. Allison J, Campbell IL, Morahan G, Mandel TE, Harrison LC, Miller JFAP. Diabetes in transgenic mice resulting from over-expression of class I histocompatibility molecules in pancreatic beta cells. Nature 1988; 333: 529-533.

10. Frauman $A$, Chu $P$, Harrisson LC. Nonimmune thyroid destruction results from transgenic overexpression of an allogeneic major histocompatibility complex class I protein. Mol Cell Biol 1993; 13: 1554-1564

11. Jaffe L, Robertson EJ, Bikoff EK. Developmental failure of chimeric embryos expressing high levels of H-2Dd transplantation antigens. Proc Nat Acad Sci 1992; 89: 5927-5931.

12. Martin WD, Egan RM, Stevens JL, Woodward JG. Lens-specific expression of a major histocompatibility complex class I molecule disrupts normal lens development and induces cataracts in transgenic mice. Invest Ophthalmol Vis Sci 1995; 36: 1144-1154.

13. Turnley AM, Morahan G, Okano H, Bernard O, Mikoshiba K, Allison J et al. Dysmyelination in transgenic mice resulting from expression of class I histocompatibility molecules in oligodendrocytes. Nature 1991; 353: 566-569.

14. Zijlstra M, Bix M, Simister NE, Loring JM, Raulet DH, Jaenisch R. Beta 2-microglobulin deficient mice lack CD4-8+ cytolytic T cells. Nature 1990; 344: 742-746.

15. Anichini A, Ress S, Strassmann G, Bach FH. Inhibition of anti-class I cytotoxicity by anticlass II monoclonal antibodies (MoAb). II. Blocking of anti-class I CTL clones by anti-DR MoAb. Hum Immunol 1985; 13: 139-143.

16. Vogelstein B, Lane D, Levine AJ. Surfing the p53 network. Nature 2000; 408: 307-310.

17. Moll UM, LaQuaglia M, Benard J, Riou G. Wild-type p53 protein undergoes cytoplasmic sequestration in undifferentiated neuroblastomas but not in differentiated tumors. Proc Natl Acad Sci USA 1995; 92: 4407-4411.

18. Qu L, Huang S, Baltzis D, Rivas-Estilla AM, Pluquet O, Hatzoglou M et al. Endoplasmic reticulum stress induces p53 cytoplasmic localization and prevents p53-dependent apoptosis by a pathway involving glycogen synthase kinase-3beta. Genes Dev 2004; 8: 261-277.

19. Li J, Lee AS. Stress induction of GRP78/BiP and its role in cancer. Curr Mol Med 2006; 6: 45-54.

20. Yoshida H, Okada T, Haze K, Yanagi H, Yura T, Negishi M et al. ATF6 activated by proteolysis binds in the presence of NF-Y (CBF) directly to the cis-acting element responsible for the mammalian unfolded protein response. Mol Cell Biol 2000; 20: $6755-6767$

21. Calfon $M$, Zeng $H$, Urano $F$, Till JH, Hubbard SR, Harding HP et al. IRE1 couples endoplasmic reticulum load to secretory capacity by processing the XBP-1 mRNA. Nature 2002; 415: 92-96.

22. Harding HP, Zhang Y, Ron D. Protein translation and folding are coupled by an endoplasmic-reticulum-resident kinase. Nature 1999; 397: 271-274.

23. Day PM, Esquivel F, Lukszo J, Bennink JR, Yewdell JW. Effect of TAP on the generation and intracellular trafficking of peptide-receptive major histocompatibility complex class I molecules. Immunity 1995; 2: 137-147.

24. Martien van Santen H, Woolsey A, Rickardt PG, Van Kaer L, Baas EJ, Berns A et al. Increase in positive selection of CD8+ T cells in TAP1-mutant mice by human beta 2microglobulin transgene. J Exp Med 1995; 181: 787-792.

25. Bernards R, Dessain SK, Weinberg RA. N-myc amplification causes down-modulation of MHC class I antigen expression in neuroblastoma. Cell 1986; 47: 667-674.

26. Yamit-Hezi A, Plaksin D, Eisenbach L. c-fos and c-jun overexpression in malignant cells reduces their tumorigenic and metastatic potential, and affects their MHC class I gene expression. Oncogene 1994; 9: 1065-1079.

27. Yuan J, Glazer PM. Mutagenesis induced by the tumor microenvironment. Mutat Res 1998; 400: $439-446$

28. Linda H, Hammarberg H, Piehl F, Khademi M, Olsson T. Expression of MHC class I heavy chain and beta2-microglobulin in rat brainstem motoneurons and nigral dopaminergic neurons. J Neuroimmunol 1999; 101: 76-86.

29. Finsen BR, Jorgensen MB, Diemer NH, Zimmer J. Microglial MHC antigen expression after ischemic and kainic acid lesions of the adult rat hippocampus. Glia 1993; 7: 41-49.

30. Watson NF, Ramage JM, Madjd Z, Spendlove I, Ellis IO, Scholefield JH et al. Immunosurveillance is active in colorectal cancer as downregulation but not complete loss of MHC class I expression correlates with a poor prognosis. Int J Cancer 2006; 118: 6-10.

31. Fernandez PM, Tabbara SO, Jacobs LK, Manning FC, Tsangaris TN, Schwartz AM et al. Overexpression of the glucose-regulated stress gene GRP78 in malignant but not benign human breast lesions. Breast Cancer Res Treat 2000; 59: 15-26. 
32. Lee E, Nichols P, Spicer D, Groshen S, Yu MC, Lee AS. GRP78 as a novel predictor of responsiveness to chemotherapy in breast cancer. Can Res 2006; 66: 7849-7853.

33. Reddy RK, Mao C, Baumeister P, Austin RC, Kaufman RJ, Lee AS. Endoplasmic reticulum chaperone protein GRP78 protects cells from apoptosis induced by topoisomerase inhibitors: role of ATP binding site in suppression of caspase-7 activation. J Biol Chem 2003; 278: 20915-20924.

34. Shuda M, Kondoh N, Imazeki N, Tanaka K, Okada T, Mori K et al. Activation of the ATF6, XBP1 and grp78 genes in human hepatocellular carcinoma: a possible involvement of the ER stress pathway in hepatocarcinogenesis. J Hepatol 2003; 38: 605-614.

35. Cullinan SB, Zhang D, Hannink M, Arvisais E, Kaufman RJ, Diehl JA. Nrf2 is a direct PERK substrate and effector of PERK-dependent cell survival. Mol Cell Biol 2003; 23 7198-7209.
36. Romero-Ramirez L, Cao H, Nelson D, Hammond $\mathrm{E}$, Lee AH, Yoshida $\mathrm{H}$ et al. XBP1 is essential for survival under hypoxic conditions and is required for tumor growth. Cance Res 2004; 64: 5943-5947.

37. Li XA, Lee AS. Competitive inhibition of a set of endoplasmic reticulum protein genes (GRP78, GRP94, and ERp72) retards cell growth and lowers viability after ionophore treatment. Mol Cell Biol 1991; 11: 3446-3453.

38. Sabapathy K, Hochedlinger K, Nam SY, Bauer A, Karin M, Wagner EF. Distinct roles for JNK1 and JNK2 in regulating JNK activity and c-Jun-dependent cell proliferation. Mol Cell 2004; 15: 713-725.

39. Lee MK, Hande MP, Sabapathy K. Ectopic mTERT expression in mouse embryonic stem cells does not affect differentiation but confers resistance to differentiation- and stressinduced p53-dependent apoptosis. J Cell Sci 2005; 118: 819-829. 